\title{
ReaR
}

ISNN 19894090

Revista electrónica de AnestesiaR

FORMACIÓN MÉDICA

Mayo 2020

\section{Acidosis propiónica, enfermedad metabólica rara con manejo anestésico peculiar.}

Rubio López JD, Muñoz Martínez R

\section{H.U. Reina Sofía de Córdoba}

\section{Resumen}

La Acidosis Propiónica es una enfermedad metabólica mitocondrial que afecta al catabolismo de los ácidos grasos, colesterol y los aminoácidos de cadena ramificada a causa de un déficit enzimático. No existe una correlación del genotipo con la severidad de la enfermedad. Es considerada como una enfermedad rara, aunque su incidencia es variable, y dentro de los errores del metabolismo es de los más frecuentes. En el contexto de un paciente con esta patología que va a requerir ser anestesiado en varias ocasiones procedemos a la revisión de la fisiopatología de la enfermedad, sus complicaciones e investigar en las implicaciones anestésicas del manejo anestésico futuro.

\section{Introducción}

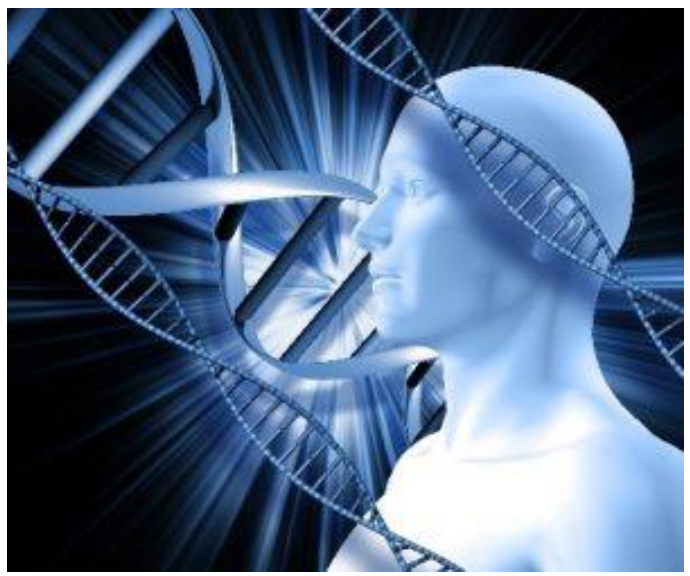

La Acidosis Propiónica es una enfermedad metabólica mitocondrial que afecta al catabolismo de los ácidos grasos, colesterol y los aminoácidos de cadena ramificada a causa de un déficit enzimático. No existe una correlación del genotipo con la severidad de la enfermedad. Es considerada como una enfermedad rara, aunque su incidencia es variable, y dentro de los errores del metabolismo es de los más frecuentes. En el contexto de un paciente con esta patología que va a requerir ser anestesiado en varias ocasiones procedemos a la revisión de la fisiopatología de la enfermedad, sus complicaciones e investigar en las implicaciones anestésicas del manejo anestésico futuro.

\section{Metodología}

Iniciamos una búsqueda bibliográfica a través de la web de PUBMED en la que se introdujeron los siguientes caracteres propionic acidemia and anesthesia, realizando así la revisión de 10 de los artículos encontrados.

También se consultó bibliografía referente a la fisiopatología, las complicaciones derivadas y el tratamiento existente para la enfermedad.

\section{Resultados}

Esta patología es resultado de una mutación genética de herencia autosómica recesiva, y radica en la deficiencia de la enzima mitocondrial Propionil - CoA carboxilasa, sin la cual 
se acumulan ácidos orgánicos característicos debido al catabolismo de los ácidos grasos de cadena impar, las cadenas laterales del colesterol, aminoácidos ramificados (Leucina, Isoleucina, Treonina, Metionina), Tiamina y Uracilo; y la fermentación bacteriana de los carbohidratos.

$\mathrm{Su}$ descompensación puede desencadenar una acidosis láctica que propicia una hiperamonemia, las cuales prolongadas en el tiempo y sin control pueden producir consecuencias negativas en distintos órganos $\mathrm{y}$ aparatos de modo irreversible e incluso la muerte. Entre las complicaciones más frecuentes asociadas destacamos la cardiomiopatía, epilepsia, retraso cognitivo y anemia - trombocitopenia, entre otros.

Existen dos situaciones fundamentales en las que nos enfrentamos a esta patología. El paciente pediátrico de corta edad en la que el diagnóstico es reciente e insidioso, y se encuentra en un momento agudo de la evolución. Y el segundo caso de una edad mayor con complicaciones ya establecidas, pero generalmente estables y bien controlados.

Ante una situación inusual nos vemos obligados a estudiar y proponer una estrategia para el óptimo manejo y control del paciente y su enfermedad. Scholtes JL y Veyckemasns (7) proponen un esquema general para las enfermedades metabólicas raras en las que resulta crucial plantear previamente las consecuencias de la enfermedad para la anestesia en materia de vía aérea, función cardíaca, antecedentes de epilepsia, la probabilidad de rabdiomiolisis o hipertermia maligna, y la fragilidad esquelética. Así como revisar su tratamiento habitual y las consideraciones de su dieta. También se valorará las consecuencias del estrés, la fiebre o el periodo perioperatorio para el equilibrio metabólico; el impacto de los agentes anestésicos sobre la homeostasis y sus secuelas sobre la enfermedad; los resultados deletéreos de la cirugía como la aspiración de restos sanguíneos o problemas respiratorios postopetarios; la necesidad de soluciones de mantenimiento, y la monitorización metabólica específica necesaria.

En el caso de la acidosis propiónica, durante su evaluación preoperatoria pondremos atención en su balance ácido - base, al estado nutricional, el tono muscular, el estado mental, la función gastrointestinal y cardíaca. Ello nos ayudará a enfocar las medidas o el manejo requeridos para evitar la hipoxia e hipertensión para mantener las óptimas condiciones de los tejidos, la deshidratación para no facilitar la acidosis, la hipoglucemia para no provocar estados catabólicos y para proponer el uso de fármacos anestésicos más aconsejados. Además, se recomendará el mantenimiento de la dieta baja en proteínas y aumentar la ingesta de carbohidratos. Se deben evitar largos períodos de ayuno, y si se requiere, mantener con soluciones intravenosas con carbohidratos y suplementos libres de sustratos propiogénicos. Seguirá con su medicación habitual de causa (Lcarnitina o glicina que le ayuda a eliminar vía renal el exceso de ácido propiónico, laxantes o metronidazol para prevenir el sobrecrecimiento bacteriano, $\mathrm{u}$ otros tratamientos administrados como el benzoato sódico, biotina o L-arginina que también influyen en el metabolismo) o de efecto (antiepilépticos, reflujo gastroesofágico $\mathrm{u}$ otros). Se solicitará un conjunto general pruebas de laboratorio preoperatorias entre las que encontramos el hemograma, una gasometría arterial, concentración de amonio, electrolitos, glucosa sanguínea, láctico sérico, aminoácidos plasmáticos, 
niveles de L-carnitina, y ácidos orgánicos en orina; entre otras que puedan ser necesarias dependiendo de las complicaciones a valorar.

Se recomienda que estos pacientes debido a su peculiaridad médica y la necesidad de cuidados sean ingresados en hospitalización el día previo a la intervención.

En la jornada de la intervención, además de las pautas anteriores, se administrará tratamiento profiláctico de reflujo gastroesofágico y se planificará una inducción de secuencia rápida por el alto riesgo de aspiración que estos enfermos padecen. En el quirófano requieren una monitorización estándar (electrocardiograma, presión arterial no invasiva, pulsioximetría, capnografía, temperatura central) valorando la canalización de una vía arterial si se prevé que el proceso sea de larga duración, para poder realizar un control gasométrico periódico, u otras medidas según la intervención quirúrgica como el acceso venoso central u otro tipo de monitorización invasiva.

El manejo de los anestésicos se hará considerando la composición de sus fórmulas, sus efectos adversos más comunes asociados y sus implicaciones metabólicas en el organismo. Se contraindica pues el uso de propofol por su contenido de ácidos grasos, mientras que el etomidato y la ketamina no son recomendados por su acción epileptógena. En cambio, parece ser seguro el Tiopental sódico, las benzodiacepinas como el midazolam, dexmedetomidina y los mórficos, así como los gases inhalatorios (sevoflurano, desflurano o isoflurano), exceptuando el óxido nitroso por su inhibición sobre el metabolismo de la vitamina B12. Independientemente de los fármacos depresores elegidos hay que tener en cuenta, la gran susceptibilidad de estos pacientes a los efectos depresivos que les provoca, resultando en una hipotonía y letargia prolongadas, por lo que se advierte ajustar sus dosis. No hay trabajos en los que apoyen ni comenten el uso de la monitorización de profundidad anestésica para el mantenimiento durante las intervenciones, por lo que se tomaría en consideración su uso para el ajuste de dosis. Los relajantes neuromusculares de la familia aminoesteroides como el rocurinio o vecuronio son los que resultan más seguros, mientras que están contraindicados aquellos en los que en su proceso de degradación exista alguna reacción de esterificación-hidrólisis como con la succinilcolina, atracurio y cisatracurio, aunque algunos en trabajos resultaran seguros a dosis bajas.

Teóricamente existe un riesgo incrementado de toxicidad sistémica con el uso de anestésicos locales por ser inhibidores de la formación de ATP mitocondrial y afectar a su metabolismo. Ellinas $\mathrm{H}$ et al. (11) proponen en su trabajo que la anestesia regional podría ser llevada a cabo en pacientes seleccionados que sufren de estas enfermedades metabólicas que afectan a la función mitocondrial. Soberón JR et al. (3) realizan una técnica ecoguiada y con ajuste de dosis de anestésico local (Ropivacaína) en un caso de acidosis propiónica, con un resultado favorable y sin incidencias. Permanece la duda de si ante un caso de toxicidad sistémica se pudiera optar por el intralipid intravenoso como medida de urgencia.

El manejo de fluidoterapia de mantenimiento intravenoso es de gran interés. Idealmente se debe evitar el catabolismo y siempre a favor de mantener la homeostasis interna. Por lo que se requiere una infusión de soluciones con glucosa o dextrosa a distintas concentraciones dependiendo de los requerimientos para cumplir una 
de las anteriores premisas. En cuanto al uso del resto de fluidos, se contraindica el uso de aquellas que contengan lactato en su composición para evitarlo como factor precipitante de desequilibrios ácido - base. Según la bibliografía consultada se han utilizado con seguridad el suero salino con cloruro sódico al $0.9 \%$ y soluciones coloidales como el hidroxetil-almidón. A día de hoy se disponen de otros sueros balanceadas como la solución Ringer sin lactato entre otras, y soluciones coloidales balanceadas por las que podrían optarse, evitando todas aquellas que en su formulación contengan algún derivado proteico como la albúmina. No existen consideraciones especiales para la transfusión de hemoderivados, solo un grupo transmite la experiencia de administración de un concentrado de hematíes durante un trasplante hepático en las que se toman medidas de gran precaución en su administración con un resultado favorable. Así bien se recomienda el uso de bicarbonato u otra formulación de reposición que así lo requiera para el tratamiento del desequilibrio osmótico interno. Tampoco existe ninguna contraindicación ni consideración al uso de drogas vasoactivas respecto a otro tipo de paciente.

Un último aspecto a tener en cuenta desde el punto de vista farmacológico es el uso de analgésicos periféricos. Están contraindicados aquellos

antiinflamatorios no estereoideos que son derivados del ácido propiónico entre los que encontramos el ibuprofeno, dexketoprofeno y naproxeno, También se advierte del uso de la meperidina debido a que el acumulo de sus metabolitos puede desencadenar convulsiones.

Dentro de las medidas recomendables durante la cirugía se aconseja la colocación de una sonda de aspiración continua en estómago en aquellas cirugías como ORL u odontología, en las que exista un riesgo de deglución de sangre.

Durante el período postoperatorio, estos pacientes tienen un riesgo aumentado de desarrollar un distress respiratorio a causa de la fatiga o la obstrucción de las vías aéreas superiores, por lo que se propone la extubación tras comprobar la recuperación de la relajación muscular. No existe experiencia en el uso de reversión del bloqueo neuromuscular en dichos pacientes por lo que sería de interés el posible efecto favorable de su administración en ellos.

Es importante el análisis de su situación clínica crítica más común, la hiperamonemia. Existe un consenso de manejo médico consensuado en la conferencia de Washington de 2011 para el tratamiento de dicha afección, en la que se recomiendan las siguientes pautas: a) Administrar sedación, ventilación y agentes vasopresores según se requiera. b) Evitar el catabolismo mediante la infusión de soluciones carbohidratadas. Optar por la hemodiálisis, hemofiltrado o ECMO en el caso de que el amonio supere los $3000 \mathrm{mmol} / \mathrm{L}$, exista una acidosis extrema, un desequilibrio electrolítico grave $o$ se encuentre en coma con pupilas dilatadas, focalidad neurológica más deterioro o fallo respiratorio. c) Se aconseja monitorizar periódicamente el amonio, electrolitos, y los gases sanguíneos, así como la realización de una monitorización neurológica continua. d) Y por último se indica la administración de bolos de L-carnitina de 100 mg durante 3-4 veces al día.

\section{Discusión}

Ninguna de estas recomendaciones está basada en la evidencia científica sino de la experiencia de distintos profesionales ante la presentación de dichos pacientes. Además, alguna de las recomendaciones 
teóricamente resulta controvertida ya que como se puede observar en la literatura hay quien actúa en contra de dichas premisas sin obtener ningún resultado desfavorable. Nuestra actuación irá encaminada siempre con el objetivo final de mantener al paciente en las mejores condiciones, valorando el riesgo - beneficio global ante una nueva decisión. Como esquema orientativo, se propone el que muestra la tabla 1.

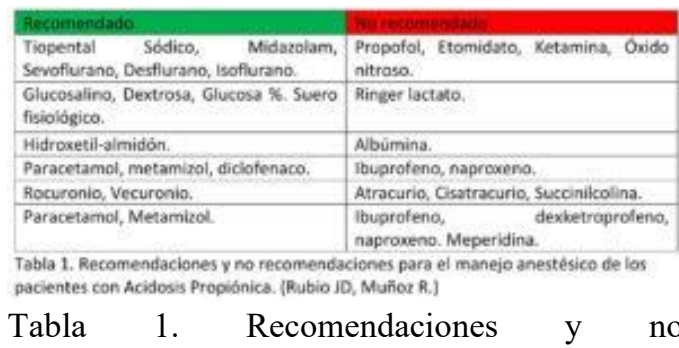
recomendaciones para el manejo anestésico de los pacientes con Acidosis Propiónica. (Rubio JD, Muñoz R.)

\section{Bibliografía}

1. Ruzkova K,Weingarten TN, Larson KJ, Friedhoff RJ, Gavrilov DK, Sprung J. Anesthesia and organic aciduria: is the use of lactated Ringer's solution absolutely contraindicated? Pediatric Anesthesia; 2015; 25: 807-817. (PubMed)

2. Arcas-bellas JJ, Arévalo-Ludeña J, Oñate ML, Aranzubia M, ÁlvarezRementería R, Muñoz-Alameda L. General anesthesia in an adult female with propionic acidemia: anesthetic considerations, Minerva Anestesiologica; 2013; 79(3); 313-315. (HTML)

3. Soberón JR,Elliott CE, Bland KS, Weinberg GL. Peripheral Nerve Block in a Patient with Propionic Acidemia, Regional Anesthesia and Pain Medicine; 2014; 39(6); 560-561. (PubMed)

4. Eugene H,Emhardt JD, Hainline BE. Propionic Acidemia in a Four-MonthOld Male: A Case Study and Anesthetic Implications; Anesthesia Analgesia; 2000; 91; 309-311 (PubMed)

5. Cammarata-Scalisi F, Yen-Hui C, TzeTze L, Da Silva G, Araque D, Callea $\mathrm{M}$ et al; Clinical, biochemical and molecular findings of propionic acidemia; Arch Argent Pediatr 2019;117(3); e288-e291. (PubMed)

6. Karagoz AH et al.; Anesthetic management of a 2-year-old male with propionic acidemia; Pediatric Anesthesia; 2006; 16; 1289-1296.

7. Scholtes et al.; The child with a known metabolic disease: Anaesthetic management; Annales Francaises d'Anesthesie et de Reanimation; 2010; $19 ; 568-569$.

8. Ryu J. Shin YH, Ko JS, Gwak MS, Kim GS. Intractable metabolic acidosis in a child with propionic acidemia undergoing liver transplantation-a case report-; Korean Journal Anesthesiology; 2013 September; 65(3); 257-261. ( $\underline{\mathrm{PMC}})$

9. Chu TH, Chien YH, Lin HY, Liao HC, Ho HJ, Lai CJ, et al.; Methylmalonic acidemia/propionic acidemia - the biochemical presentation and comparing the outcome between liver transplantation versus non-liver transplantation groups; Orphanet Journal of Rare Diseases; 2019; 14; 73. (PubMed)

10. Rajakumar A, Kaliamoorthy I, Reddy MS, Rela M. Anaesthetic considerations for liver transplantation in propionic acidemia. Indian Journal Anaesthesia; 2016; 60; 50-54. (HTML)

11. Ellinas H, Frost EA. Mitochondrial disorders - a review of anesthetic considerations. MiddleEast J Anesthesiol. 2011; 21; 235-242. (DF)

12. Haijes HA, Jans JJM, Tas SY, Verhoeven-Duif NM, van Hasselt PM et al. Pathophysiology of propionic and methylmalonic acidemias. Part 1: Complications; J Inherit Metab Dis. 2019 Sep;42(5):730-744.(PubMed)

13. Haijes HA, van Hasselt PM, Jans JJM, Verhoeven-Duif NM, et al. Pathophysiology of propionic and methylmalonic acidemias. Part 2: treatment strategies; J Inherit Metab Dis.; $2019 \quad$ Sep;42(5):745-761. (PubMed) 
Correspondencia al autor

Jesús David Rubio López

jesusdavidrubiolopez@gmail.com

Residente Anestesiología y Reanimación.

H.U. Reina Sofía de Córdoba
Rafael Muñoz Martínez

FEA Anestesiología y Reanimación.

H.U. Reina Sofía de Córdoba.

Aceptado para el blog en noviembre de 2019 